\title{
Der Algorithmus, meine Arbeit und Ich - Einfluss von Algorithmen auf Content Creator:innen der Video Plattform YouTube
}

\author{
Milena Albiez ${ }^{1}$
}

Angenommen: 9. Dezember 2021 / Online publiziert: 24. Januar 2022

(c) Der/die Autor(en) 2022

\section{Zusammenfassung}

Dieser Beitrag in der Zeitschrift „Gruppe. Interaktion. Organisation. (GIO)“ nimmt auf den Einfluss von Algorithmen der Video-Plattform YouTube auf den Arbeitsalltag von Content-Creator:innen Bezug. Für den Aufbau eines erfolgreichen, also Abonnent:innen-starken Kanals und einer beruflichen Tätigkeit als Content-Creator:in, müssen YouTuber:innen sich der Wirkweisen der eingesetzten Algorithmen bewusst sein. Es wird dargestellt, welchen Einfluss Spracherkennungsund Analysealgorithmen auf die Sprache und das Verhalten vor der Kamera haben und welcher Logik die von YouTube eingesetzten Algorithmen zu folgen scheinen. Haupt- und nebenberufliche YouTuber:innen sind von YouTube, Änderungen in den Wirkweisen der Algorithmen und technischen Störungen in gewisser Weise abhängig. Diese Abhängigkeit und gefühlte Machtlosigkeit kann unter bestimmten Umständen zu einer übermäßigen beruflichen Belastung führen und eine Ursache für einen Burnout sein. Im Widerspruch zur Abhängigkeit wird die Tätigkeit als Content Creator:in gleichzeitig auch als große Freiheit wahrgenommen.

Schlüsselwörter Social Media · Coaching · Organisation · YouTube · Digitalisierung · YouTuber · Content Creator · Selbstständigkeit $\cdot$ Google $\cdot$ Burnout $\cdot$ Stress $\cdot$ New Work

\section{The algorithm, my work and me-influence of algorithms on content creators on the video platform YouTube}

\begin{abstract}
This article in the journal "Gruppe, Interaktion, Organisation (GIO)" ("Group, Interaction and Organization") refers to the influence of algorithms, as applied by the video-platform YouTube, on the daily work of content creators. In order to build up a successful channel, attracting many subscribers, content creators have to be aware of the mechanisms underlying the algorithms applied. Here, we exemplify how algorithms programmed for voice recognition and analysis influence their linguistic behavior and their acting in front of a camera. What is the rationale of algorithms which seems to be pursued by YouTube? Part-time and professional YouTubers are dependent on changes in the modes of action and possible disturbances of the algorithms. These dependences and felt incapacity may-under certain circumstances-lead to an excessive stress situation and thereby even to a burn-out. At the same time and in contradiction to these dependences, the activity of a content creator is sometimes experienced as a higher degree of freedom.
\end{abstract}

Keywords Social media - Coaching - Organization · YouTube - Digitalization · YouTuber · Content creator · Self-employed $\cdot$ Google $\cdot$ Burnout $\cdot$ Stress $\cdot$ New work

Milena Albiez, M.F.A.

hallo@milenaalbiez.de, albiez@uni-kassel.de

1 Universität Kassel, Kassel, Deutschland 


\section{Einleitung}

Die Digitalisierung ${ }^{1}$ ist eine Veränderung, welche vor mehr als 40 Jahren begonnen hat und ,es scheint als würden wir mit der Digitalisierung in eine zweite Phase der Aufklärung und Emanzipation eintreten" (Bunz 2012, S. 133). Dass diese Veränderungen auch die Arbeitswelt betreffen, ist evident. Welchen Einfluss hat die Digitalisierung auf Personen, die sich in ihrer Arbeit zwischen virtuellen und physischen Welten bewegen? Inwiefern sind YouTuber:innen abhängig von der Videoplattform YouTube, deren vorgegebenen oder landesspezifischen Richtlinien, den Algorithmen und Systematiken, die die hochgeladenen Videos präsentieren? Wie wirkt sich diese Abhängigkeit auf das Arbeitsfeld aus?

Im GIO-Artikel „Systemische, soziale und emotionale Herausforderungen und Glücksmomente im beruflichen Alltag von haupt- und nebenberuflichen YouTuber*innen" (Albiez 2020) habe ich bereits unterschiedliche Belastungen beschrieben, mit denen YouTuber:innen in ihrer Arbeit konfrontiert sein können. Die Ursachen dieser Belastungen liegen auch unter anderem in systemimmanenten Strukturen wie z.B. den Auswirkungen von Algorithmen und Community-Guidelines ${ }^{2}$, auf die YouTuber*innen als Einzelpersonen wenig Einfluss nehmen können. Dem gegenüber stehen jedoch übermäßig positiv beschriebene Glücksmomente, die dazu führen, dass diese Personen die Tätigkeit weiterführen. Dieser Artikel soll die Ursachen ebendieser systemimmanenten Auswirkungen weiter ausführen, Widersprüche aufzeigen und den Einfluss von YouTube als Organisation auf die YouTuber:innen deutlich machen.

Kelly Svirakova alias Kelly MissesVlog eine deutschsprachige YouTuberin mit ca. 1,95 Mio Abonnent:innen ${ }^{3}$ sagt in ihrem Video „Meine YouTube Karriere ist zu Ende“4 folgendes: „Ich hab schon immer gewusst, dass ich von dieser Plattform YouTube extrem abhängig bin und wenn die einen nicht mehr auf dem Schirm haben wollen, dann bist du einfach abgeschossen. Wenn denen irgendein Fehler unterläuft, hast du verkackt.“. Der hier vorliegende Artikel hat zum Ziel, den Umfang des Einflusses von Algorithmen auf YouTuber:innen zu beschreiben, dies zu hinterfragen, jedoch nicht ihren Einsatz generell auf YouTube als dysto-

\footnotetext{
${ }^{1}$ unter Digitalisierung ist hier der Einsatz digitaler Technologien gemeint, die dazu genutzt wird, ein Geschäftsmodell in digitale oder virtuelle Räume zu transformieren. Damit gehen neue Umsatz- und Wertschöpfungsmöglichkeiten oder auch ein Zugzwang von Geschäftsmodellen sich unter veränderten Marktbedingungen oder Zielgruppen zu entwickeln einher.

$2<$ https://www.youtube.com/intl/de/yt/about/policies/\#communityguidelines $>$ [12.04.2019].

3 Stand 03.08.2021.

${ }^{4}$ Kelly MissesVlog: „Meine YouTube Karriere ist zu Ende.“ vom 07.12.2016 <https://www.youtube.com/watch?v=qWdp8enl-pw> [15.08.2021].
}

pisches Szenario darzustellen. Die Behauptung „Social-Media macht Menschen zu Ich-bezogenen Vollidioten“ könnte in 20 Jahren genau so peinlich sein, wie die Diskussion der American Newspaper Publishers' Association 1897, ob Schreibmaschinen die literarische Qualität von Journalisten senkten (Passig 2013). Genauso naiv wäre es, den Einfluss von Technik, Medien und Prozessen und das damit einhergehende Tempo auf das Individuum und die Gesellschaft zu negieren.

\section{YouTube als Teil der Alphabet LCC}

Die YouTube LLC ist eine Tochtergesellschaft der Google LLC, welche seit 2015 Teil der Alphabet Inc. Holding ist. Neben dem für die gleichnamige Suchmaschine bekannten Unternehmen Google umfasst Alphabet weitere Unternehmen wie Waymo (Entwicklung autonomer Fahrzeuge - Google Driverless Car), DeepMind (Künstliche Intelligenz - AlphaGo erstes Programm, welches unter Wettkampfregeln professionelle Go-Spieler besiegte), $\mathrm{Ca}$ lico (Biotechnologie, Forschung gegen menschliche Alterung), GoogleCapital und GoogleVenture (Finanzierung und Risikokapitalgeber) und andere Technologie-Unternehmen. Die Google LLC stellt seinen Nutzer:innen neben YouTube unterschiedliche Dienstleistungen zur Verfügung wie z.B. Suchmaschine, Werbedienstleistungen (Google Ads, Google AdSense), Karten (Maps, StreetView, Earth), Kalender und Datenspeicherung (Drive), Kommunikation (Gmail, GoogleHangout, Google+) und das Betriebssystem Android. Außerdem bietet es unterschiedliche HardwareProdukte und Smart Home Devices an. Google Ads und AdSense sind u.a. zwei Google Dienstleistungen, die mit YouTube direkt verknüpft sind und auf welche in späteren Abschnitten noch eingegangen wird. Auch die Einflüsse von künstlicher Intelligenz, Sprach- oder Bilderkennungsalgorithmen, die in anderen Alphabet-Unternehmen oder bei Google entwickelt wurden und werden, kommen vermutlich bei YouTube zum Einsatz. So bietet YouTube mittlerweile automatische Untertitel bei Videos an. Diese werden über einen Spracherkennungsalgorithmus direkt in Untertitel in die der jeweiligen ausgewählten Sprache umgewandelt. Diese Algorithmen müssen mithilfe von gesammelten Datensätzen eingelernt werden, die u.a. aus der Spracheingabe von Google Voice Search stammen, die User getätigt haben ${ }^{5}$. Somit betätigen sich User indirekt am Einlernen der Algorithmen und künstlichen Intelligenz. Der Leserschaft sind eventuell sogenannte Captchas ein Begriff,

\footnotetext{
5 vgl. Alberti, Christopher und Bacchiani, Michiel: „Automatic Captioning in YouTube" vom 04.12.2009.

$<$ https://ai.googleblog.com/2009/12/automatic-captioning-inyoutube.html $>$ [19.08.2021]
} 
kleine aufpoppende Fenster, in welchen der Internet-User seine Menschlichkeit demonstrieren soll, indem er oder sie bestimmte verschwommene Zahlen und Buchstabenkombinationen eingeben oder Busse, Ampeln oder andere Bildelemente erkennen soll. Die Datensätze, die sich aus letzterem Beispiel ergeben, werden bei der Bilderkennung von Google Maps oder Google StreetView genutzt ${ }^{6}$. Bilderkennungsalgorithmen werden bei der Kategorisierung von Videos eingesetzt ${ }^{7}$. Das heißt ein Algorithmus kann erkennen, welche Bildelemente in einem Video zu sehen sind und versieht diese mit einer Art Label. In der Nutzung und Verschränkung der Technologie ineinander wird ersichtlich, dass YouTube zwar hinsichtlich der Organisation eigenständig betrachtet werden kann, jedoch inhaltlich und technologisch mit anderen Bereichen von Alphabet dicht verwoben ist.

\section{Tätigkeit als Content Creator:in auf YouTube}

Als Content Creator:in auf YouTube oder YouTuber:in werden auf YouTube Personen bezeichnet, die Videoinhalte für einen Kanal konzipieren, produzieren und oder moderieren (Burgess und Green 2009). Der deutschsprachige YouTubeStandort in Deutschland, Österreich und der Schweiz ist für deutschsprachige-YouTuber:innen aufgrund der demografischen Faktoren und potenziellen Zielgruppen limitiert. Englischsprachige YouTuber:innen erreichen z. B. international viel größere Zielgruppen.

Um haupt- oder nebenberuflich mit Content auf YouTube Geld zu verdienen, müssen Personen unter bestimmten Voraussetzungen in das YouTube-Partnerprogramm ${ }^{8}$ aufgenommen werden ${ }^{9}$. Nach einer erfolgreichen Bewerbung kann über das YouTube-Partnerprogramm Geld verdient werden. Die gängigste Einkommensquelle ist die Beteiligung an Werbeeinnahmen, in dem neben oder in dem Video Werbeinhalte gezeigt werden ${ }^{10}$. YouTuber:innen müssen sich an die von Google festgelegten Richtlinien für

\footnotetext{
6 vgl. Ibarz, Julian und Banerjee, Sujoy: „Updating Google Maps with Deep Learning and Street View“ vom 03.05.2017<https://ai. googleblog.com/2017/05/updating-google-maps-with-deep-learning. html $>$ [19.08.2021].

7 vgl. Natsev, Paul: „An updated YouTube-8M, a video understanding challenge, and a CVPR workshop. Oh my!“ vom 15.02.2017 $<$ https://ai.googleblog.com/2017/02/an-updated-youtube-8m-video. html $>$ [19.08.2020].

$8<$ https://support.google.com/youtube/answer/72851?hl=de > [15.11.2021].

9 <https://support.google.com/youtube/answer/72851\#reviewprocess> [15.08.2021].

$10<\mathrm{https}: / /$ support.google.com/youtube/answer/2467968> [19.08.2021].
}

werbefreundliche Inhalte ${ }^{11}$ halten, damit ihre Videos für das Schalten von Werbung in Frage kommen, also monetarisiert werden können. Für die Schaltung von Werbeinhalten müssen Creator ein Google AdSense Konto haben. AdSense ${ }^{12}$ ist ein Online-Dienst von Google der mit Algorithmen passende Werbung für User bereitstellt. Mit AdSense ist auch GoogleAds ${ }^{13}$ verknüpft, wo Kunden festlegen können, mit welchen Anteilen Webseiten oder Accounts, auf denen Werbung geschaltet wird, für das Schalten von Werbung entlohnt werden. GoogleAds fungiert als eine Art Mittler zwischen Werbetreibenden und Webseitenbetreibenden. Außerdem können YouTuber:innen auch in Videos und anderen Social-Media Kanälen Werbung für Organisationen, Marken, Produkte oder Dienstleistungen machen, die dann monetär, über Werbeverträge, oder Affiliate Programme ${ }^{14}$ (Walsh et al. 2020), oder auch mit kostenfreien Produkten entlohnt werden. Diese Art der Einnahmen bildet einen lukrativeren Anteil für YouTuber:innen, da die Einnahmen über GoogleAds keine stabile und ausreichende Einkommensquelle sind.

\section{YouTube-Algorithmen und ihre Wirkweisen}

Die Funktions- und Wirkungsweise von YouTube-Algorithmen sind im Gegensatz zu anderen Open-Source ${ }^{15}$-Plattformen nicht für die Allgemeinheit einsehbar. Jedoch sind die Funktionsweisen dem Fachpublikum bekannt und einige Entwickler:innen veröffentlichen ihre Forschungen und Entwicklungen auf dem Google AI Blog ${ }^{16}$ und in Fachzeitschriften. Was im Detail hinter den Algorithmen von YouTube steckt, kann hier nicht vollends dargelegt werden und wird in dieser Untersuchung sekundär betrachtet. Die Algorithmen werden in der Fachliteratur ausführlich beleuchtet und zumindest ihre Wirkungsweisen beschrieben.

\subsection{Suchen und Sortieren mit Algorithmen}

Mit dem Erfolg von YouTube seit 2005 ist auch der Umfang von hochgeladenen Videos stets gestiegen und die millio-

\footnotetext{
$11<$ https://support.google.com/youtube/answer/6162278?hl=de\& ref_topic $=9285925>$ [15.08.2021].

$12<$ https://www.google.de/adsense/start/ [19.08.2021].

$13<$ https://ads.google.com/home/> [19.08.2021].

14 Als Affiliate Programm wird eine Vertriebsprovision am Verkaufserlös bezeichnet, wenn z. B. YouTuber:innen ein Produkt in einem Video vorstellen oder einen Rabattcode zur Verfügung stellen.

15 Open Source wird als Bezeichnung für Software genutzt, deren Quelltext und Programmierung öffentlich ist. Sie kann von allen eingesehen, genutzt und geändert werden und ist meistens für die User kostenlos.

$16<$ https://ai.googleblog.com/ > [19.08.2021].
} 
nenfachen Aufrufe, waren bereits nach kurzer Zeit nicht mehr zu regulieren (Marek 2013). Das heißt, dass die Menge an verfügbarem Videomaterial, wie in einem Archiv, nach einem bestimmten System geordnet werden muss, um den Usern, genau das Video anzubieten, nach dem sie suchen, um diese möglichst lang auf der Webseite zu halten. Durch die personalisierte Startseite werden jedem User bestimmte Videos vorgeschlagen, die den Interessen, welche aus alten Suchanfragen, Klick-Verhalten und geschauten Videos hervorgehen, entsprechen. Die Sortierung der Videos erfolgt durch das automatisierte Einordnen der Videos in ein Ranking. Aus der Datenbank, in welche Videos auf YouTube eingeordnet werden, wählen der Nutzer:innen aus, wird so Teil der Systemauswahllogik und beeinflusst damit die weitere Rezeption des Videos. Ein Video, das besonders oft angeschaut wird, wird mit wahrscheinlich öfter wiederholt abgerufen, was die Bedeutung des Videos verstärkt (vgl. ebd).

The entanglement of algorithmic work in the attribution of relevance is clearly visible: the arrangement in the form of ranked lists reinforces the idea that some contents deserve more prominence than others. Through the visibility given to metadata such as view, like and comment counts, this competitive process is extended throughout the platform and becomes an influential element in information diffusion as users take such indicators into account when viewing and sharing. (Rieder et al. 2018, S. 52).

Die Beteiligung der Nutzer:innen und die sich mit der Zeit daraus herausbildenden Rankings sind ein wesentlicher Teil der YouTube Algorithmen. Hier zeigt sich laut Elsaesser ein Paradox: ,the structured contingency is, on the one hand, strongly informed and shaped by mathematics, via the site's programming architecture and design, based on its search and sort algorithms. [...] the chaos of human creativity, eccentricity and self-importance prevails." (Elsaesser 2009, S. 181). Die Strukturierung der Daten/Videos erfolgt nach bestimmten Mechanismen, die bestimmte Videos oben und andere weiter unten in ein Ranking einsortiert und es gibt somit eine Diskrepanz im „Wert“ (Rankinghöhe) der Videos.

Einen kurzen Exkurs zu einem anderen Unternehmen, das Ranking-Mechanismen nutzt, möchte ich mir an dieser Stelle nicht nehmen lassen, da es die Wirkung auf den Menschen deutlich macht. Auf der Online-Dating-Plattform Tinder werden Profile der User auch in Rankings eingeordnet. Profile, die von vielen Usern, anhand der Bilder positiv bewertet wurden haben ein höheres Ranking. Auch wenn das eigene Profil von Usern mit hohen Rankings positiv bewertet wird, erhöht dies das Ranking. Profile mit hohem Ranking werden wiederum vermehrt Profilen mit hohem Ranking angezeigt. Im Klartext: Attraktive sehen Attrakti- ve und Hässliche sehen Hässliche. ${ }^{17}$ Das heißt das Ranking bestimmt eine Art Selektionsvorgang und ermittelt auch, was ein User attraktiv an anderen findet. Personen, in deren eigenem Profilbild z. B. einen Hund zu sehen ist, werden vermutlich auch mehr Personen mit Hunden vorgeschlagen. Ist ein/e vermeintliche/r Hundebesitzer:in überhaupt an anderen vermeintlichen Hundebesitzer:innen interessiert? In der Regel kann diese Frage wohl mit ja beantwortet werden, aber was ist mit den Ausnahmen dieser Regel? Was ist mit dem Zufall? Kann einer an klassischer Musik interessierten Person auf YouTube, jemals ein Video von Techno oder Punk vorgeschlagen werden? Inwiefern durch Rankingmechanismen Horizonte erweitert werden, kann durchaus angezweifelt werden.

Neben dem Klick ist mittlerweile die Watch-Time, also die Zeit, die User das Video tatsächlich ansehen, ein wichtiger Faktor geworden. So kann ermittelt werden, ob das Video mit dem Thumbnail ${ }^{18}$ und dem Titel, den Erwartungshaltungen der Zuschauer:innen entsprochen hat.

\subsubsection{Auswirkung von Interaktion auf das Ranking eines Videos}

Hohe Aktivität in den Kommentaren, das Klicken eines „Daumen-Hoch“ unter einem Video kann die Sichtbarkeit des Videos erhöhen und wird zum Beispiel mit gezieltem Fragenstellen und dem Wunsch nach Beantwortung in den Kommentaren von YouTuber:innen gesteigert. (Opresnik und Yilmaz 2016). Die Auffindbarkeit von Videos findet u. a. in den personalisierten Videovorschlägen auf der Startseite oder links neben einem Video statt. Je „beliebter“ ein Video, desto häufiger wird es bestimmten Personengruppen mit ähnlichen oder gleichen Interessen vorgeschlagen.

\subsection{Auswirkungen von Spracherkennungsalgorithmen}

Auch Spracherkennungs- und -analysealgorithmen kommen z.B. bei der Beurteilung der Werbefreundlichkeit eines Videos zum Einsatz. Werden in einem Video zu viele Schimpfwörter verwendet, wird das Video als werbeunfreundlich oder als nicht family-friendly eingeschätzt, und somit kann weniger oder keine Werbung geschaltet werden. Das heißt, dass YouTuber:innen ihre Videos durchsehen und mitunter bestimmte Wörter aus dem Material herausschneiden oder auspiepen müssen. Dieses Phänomen wird

\footnotetext{
17 vgl. Carr, Austin: „I Found Out My Secret Internal Tinder Rating And Now I Wish I Hadn't“" vom 01.11.2016 < https://www. fastcompany.com/3054871/whats-your-tinder-score-inside-the-appsinternal-ranking-system> [26.04.2019].

18 Thumbnail: dt. Vorschaubild, welches zuerst vor dem Start eines Videos angezeigt wird. Dies kann ein Videostill oder ein bearbeitetes Bild sein.
} 
auch von YouTuber:innen in Videos thematisiert. „I started to clean up my content. I started to take out anything that I thought would be demonetizeable or uuhm I don't know. I just got really scared. [...] I got really scared that I was gonna upload a video that would ruin everything. ", sagt der YouTuber Bobby Burns in einem Video ${ }^{19}$ über den eigenen Nervenzusammenbruch. YouTube folgt als amerikanisches Unternehmen den Vorschriften der amerikanischen Medienaufsichtsbehörden. Die Medien-Zensur durch z.B. Verpixeln von weiblichen Brüsten oder dem Auspiepen von Schimpfwörtern verstößt selbst nach einem Urteil eines Berufungsgerichts in New York gegen das Verfassungsrecht auf freie Meinungsäußerung, und die Bestrafung von Verstößen wird von Kritiker:innen als willkürlich bezeichnet ${ }^{20}$. Auch wenn es in Deutschland einen viel liberaleren Umgang mit Themen wie Nacktheit, Sexualität, weibliche Brust, vulgärer Sprache in den Medien gibt, müssen sich Content Creator:innen an die Regeln von YouTube halten.

Wenn ein Video als vom System automatisch als werbeunfreundlich gekennzeichnet wird oder es gegen das Urheberrecht verstößt, es jedoch keine offensichtlichen Verstöße gegen die Richtlinien gibt, können YouTuber:innen eine manuelle Überprüfung ${ }^{21}$ des Videos anfordern, welche bis zu 7 Tage in Anspruch nimmt.

\subsection{Auswirkungen von technischen Störungen}

Wenn in bestimmten Bereichen von YouTube z. B. beim Upload der Videos, in der Kommentarfunktion oder der Sichtbarkeit eines Videos in der Abobox der Abonnent:innen technische Störungen auftreten, kann das Konsequenzen für die Creator haben. So können z. B. mit Werbepartnern vereinbarte Inhalte nicht pünktlich hochgeladen oder die gängigen Aufrufzahlen nicht erreicht werden, was mitunter finanzielle Verluste mit sich bringen kann. Hier steht den YouTuber:innen zwar ein SupportTeam zur Seite, welches sich um eine zügige Lösung des Problems kümmert oder dieses an die zuständigen Stellen weiterleitet, aber das Beheben solcher Systemfehler kann viel Zeit in Anspruch nehmen, was sich negativ auf die Performance eines Videos auswirken kann.

Fest steht, dass eine Abhängigkeit der YouTuber:innen von den Mechanismen und Wirkungsweisen der Algorith-

\footnotetext{
19 Burns, Bobby: „I Had A Breakdown (Mental Health On YouTube)“ vom 04.05.2018<https://www.youtube.com/watch?v=142yMJpC32o> [26.04.2019].

${ }^{20}$ Klüver, Reymer: „Amtlich verordnete Prüderie“, Süddeutsche Zeitung Online vom 15.07.2010<https://www.sueddeutsche.de/kultur/ schimpfwoerter-im-tv-amtlich-verordnete-pruederie-1.975135>.

$21<$ https://support.google.com/youtube/answer/7083671> [19.08.2021].
}

men existiert. Ob die Abhängigkeit als eine solche empfunden wird, wird im Abschn. 5 und 6 behandelt.

Yet since the user depends on the "machine“ to generate the access points, by way of sort algorithms and tag clouds [...], a new ,authority“ interposes itself, both „stupid“ like chance and ,all-knowing“ like God. (Elsaesser 2009, S. 180f).

\subsection{Kritik an Algorithmen}

Algorithmen, Software und Technik sind, wie bereits zahlreiche Autor:innen dargestellt haben, niemals neutral, vorurteilsfrei oder unparteiisch, sondern reproduzieren im Gegenteil rassistische, sexistische, klassistische und andere diskriminierende Stereotypen der Gesellschaft (Chun 2016). Im Juni 2020 veröffentlichte die auf YouTube erfolgreiche Familie Zontay ein Video ${ }^{22}$ darüber, dass ihre Inhalte, welche sie als Schwarze Familie auf der YouTube Kids App veröffentlichten, plötzlich nicht mehr sichtbar waren. Sie kritisieren darin, dass Inhalte von und über Schwarze Menschen deutlich weniger repräsentiert werden, als die von und mit weißen Protagonist:innen. YouTube wies die Vorwürfe zurück.

\section{Auswirkungen der Algorithmen auf die Arbeit von YouTuber:innen}

Wie wirken sich nun die Mechanismen der YouTube Algorithmen auf die Personen aus, die auf YouTube Videos hochladen und als Content Creator tätig sind? YouTuber:innen müssen verstehen, wie die Mechanismen auf YouTube funktionieren, um mit ihnen zu arbeiten und den eigenen Kanal gegebenenfalls anzupassen. 2016 wurden vor allem Suchalgorithmen auf YouTube umstrukturiert. Seitdem steht das Deep Learning, also das maschinelle Lernen der Algorithmen, beim Suchen von Zuschauern nach bestimmtem Content im Vordergrund. Die Watch History, Search History und andere demografische Informationen der Zuschauer:innen werden gesammelt und analysiert. Welche Videos einer Person vorgeschlagen werden, hängt auch davon ab, was anderen Nutzer:innen vorgeschlagen wird, die ähnliche „Vorlieben“ haben. Creator sollten mindestens 2-3 Videos pro Woche mit mehr als $10 \mathrm{~min}$ produzieren, um mit „dem neuen Algorithmus“ erfolgreich zu sein (Krachten und Hengholt 2018). Das heißt, dass vor allem YouTuber:innen, deren Videos vermutlich niedrigere Rankings haben, da sie von weniger Personen abonniert

\footnotetext{
${ }^{22}$ ZZ Kids TV,„Does Black Entertainment Matters? (Our Family Story and The Youtube Kids App)“ <https://www.youtube.com/watch? $\mathrm{v}=$ gcyFHigKZPM $>$.
} 
sind, mehr Videos produzieren müssen, wenn sie gerne mehr Abonnenten oder Aufrufzahlen haben wollen. Die Regelmäßigkeit beim Upload eines Videos soll das Verbleiben an einer Stelle im Ranking oder langsames Aufsteigen im Ranking garantieren. Nach Änderungen in den Funktionsweisen der Algorithmen wurden von YouTuber:innen deutlich längere Videos hochgeladen, die mit einem entsprechend größeren Produktionsaufwand verbunden sind. Hier können nun auch mehr Werbeunterbrechungen oder Einblendungen platziert werden.

\subsection{Thematisierung der Algorithmen in Videos}

Mit der oben genannten Änderung der Algorithmen waren vor allem Kanäle von YouTuber:innen mit Personen-zentrierten Inhalten von Einbrüchen der Aufrufzahlen betroffen, welche auch in Beschwerdevideos thematisiert wurden. „Leute die mich abonniert haben, kriegen keine Benachrichtigungen mehr, oder wenn dann super viel später. Die Videos werden nicht in der Abobox angezeigt. Dazu habe ich ein paar Umfragen auf Twitter gemacht und das sind jedes Mal 30-40\% und das ist nur Twitter. Das ist nur 500.000 von den Leuten, die eigentlich hier abonniert sind. Von 1,5 Mio., also ich will gar nicht wissen, wie die Reallife Statistik aussieht. Sobald ich ein Video hochgeladen, INSTANT 200 Abos direkt gelöscht, direkt weg, niemand weiß was passiert. Ich traue mich nicht mal mehr ein Video hochzuladen [...]. " beschreibt Kelly Svirakova alias Kelly MissesVlog in ihrem bereits eingangs erwähnten Video „,Meine YouTube Karriere ist zu Ende“"23. Ihre Zugriffsquellen, also von wo Zuschauer:innen auf ihr Video stoßen, stammen laut eigenen Aussagen aus der Statistik ihres Kanals über $63 \%$ aus Videovorschlägen links neben einem Video. Der Einfluss der Algorithmen oder der Einfluss der Änderungen sind bei den Nutzer:innen und YouTuber:innen bekannt. ,Und jetzt wird einem so schmerzhaft bewusst, dass man nicht selber den Content auf YouTube steuert, sondern, dass der YouTube-Algorithmus und YouTube selber den Content auf dieser Seite steuert. Das ist jetzt wirklich schmerzhaft zu sehen.", sagt Svirakova weiter. Sie beendet das Video, indem sie ihren treuen Zuschauer:innen dankt und sich sicher ist, dass sie diese Zeit durchstehen wird. „Thanks for keeping me alive. Ich kann es nicht anders sagen, danke dass ihr mich am Leben haltet, okay? Weil ohne diese Videos, gibt es nur sehr wenig in meinem Leben, dass mich froh und munter macht, okay? Das ist einfach alles was ich habe und ich will es nicht verlieren. (seufzt). Ja. “. Inwiefern hier eine Verlustangst bei Svirakova tatsächlich vorhanden ist oder ob diese nur für den Erfolg des Videos thematisiert wurde, kann nicht be-

${ }^{23}$ Kelly MissesVlog: „Meine YouTube Karriere ist zu Ende.“ vom 07.12.2016<https://www.youtube.com/watch?v=qWdp8enl-pw> [21.04.2019]. legt werden. In ihrem Video wendet sie sich vorwiegend an ihre Zuschauer:innen, legt ihnen ihre Sicht der Dinge dar und welchen Einfluss die Änderungen am Algorithmus für ihre Videos und ihre Zuschauer:innen haben. Eine Kritik an YouTube oder ein direkter Appell zur Änderung bestimmter Wirkungsweisen findet eher versteckt als offensichtlich statt.

\subsection{Widerspruch von Freiheit und Abhängigkeit}

In der diesem Artikel zu Grunde liegenden Untersuchung (Albiez 2020) richtete sich u.a. der Blick auf die Arbeitsform der Selbständigkeit, die drei der befragten YouTuber:innen hauptberuflich ausführen. Alle befragten Personen äußerten sich positiv über ihre Freiheiten und die Unabhängigkeit, die sie in ihrer Tätigkeit als Content Creator haben. Sie benannten jedoch Schwierigkeiten im Zeitmanagement und der Work-Life-Balance, sowohl die Abhängigkeit von den Funktionen und Wirkungsweisen der Algorithmen betreffend als auch deren Herausforderungen.

Dieses Paradox wird von Muckenhuber als „Autonomieparadox" beschrieben (Muckenhuber 2014, S. 195ff). In der durchgeführten Untersuchung mit Selbstständigen wurde ein Spannungsfeld zwischen der freien Verfügbarkeit der Zeit und den realen Anforderungen der Tätigkeit sichtbar. Eine von Muckenhuber befragte Person gab an, dass ihr allein schon der Gedanke theoretisch zwei Tage frei machen zu können reiche, obwohl dies in ihrem Arbeitsalltag nie möglich sei (vgl. ebd.).

Selbstständig tätige Personen sind für ihr Zeitmanagement, Aufgabenmanagement und für ihren Erfolg selbst verantwortlich. Inwiefern Selbstständige selbst und ständig arbeiten, soll hier nicht weiter ausgeführt werden, jedoch berichteten die zwei befragten YouTuber:innen von Erschöpfungszuständen und Burnout-Symptomatiken. Ein Befragter arbeitet zusätzlich zu seinem 40-Stunden-Job noch ca. $20 \mathrm{~h}$ an seinen YouTube Videos. Der Faktor Zeit wurde von allen drei als maßgeblich für die Überarbeitung genannt. In selbstständiger Tätigkeit greifen Überstundenregelungen, Krankheits- oder Schwangerschaftsausfälle und andere Arbeitszeitmodelle nicht, um die Erwerbstätigen zu entlasten. In der erwähnten Untersuchung wurde das Paradox der empfundenen Freiheit, z.B. der Zeiteinteilung und der tatsächlichen Arbeitszeiten aufgezeigt.

Auch in meiner Untersuchung traten drei Widersprüche zu Tage, die ein Spannungsfeld der persönlichen Freiheit und den durch das System bewirkten Abhängigkeiten eröffneten:

Zum einen die im System gleichsam verankerte Unabhängigkeit und Abhängigkeit, denn YouTuber:innen sind z.B. in der Gestaltung der Videos frei, sind jedoch gleichzeitig von der Plattform YouTube und z. B. Änderungen der Algorithmen abhängig. Zum zweiten: Die vom System in 
ihren Leitsäulen ${ }^{24}$ erwähnten Freiheiten, wie z. B. die Wichtigkeit von Meinungsfreiheit für YouTube als Organisation einerseits und dem Reglement, in dem YouTuber:innen darauf achten müssen, dass nicht zu viele Schimpfwörter oder Kraftausdrücke im Video vorkommen, andererseits.

Zum dritten wurde über den Widerspruch der eigenen Ansprüche oder Ideen für Videos und die damit von Zeit zu Zeit konträren Wünsche oder Ansprüche der Zuschauer:innen gesprochen. Zwischen der Vorstellung von Freiheit und den tatsächlichen Möglichkeiten der YouTuber:innen in verschiedenen Bereichen, ist ein deutlicher Widerspruch vorhanden.

\section{Verantwortung und Einfluss von YouTube als Organisation auf YouTuber:innen}

Ein interessantes Phänomen, welches aus den Ergebnissen der Untersuchung hervorging ist, dass zwei YouTuber:innen mit unter 10.000 Abonnenten am meisten Kritik am System und der Organisation YouTube äußerten. Sie verknüpften ihre Herausforderungen direkt mit den von YouTube gestalteten Mechanismen oder Algorithmen. Wohingegen die beiden hauptberuflichen YouTuberinnen dahingehend wenig Kritik an YouTube äußerten. An dieser Stelle wäre es unbedacht, das System YouTube als nicht Teil der Vereinzelungstendenz in einem größeren gesellschaftlichen Zusammenhang zu betrachten. Ein befragter YouTuber findet, dass es vermessen sei, YouTube an dieser Stelle zu kritisieren, denn YouTube wäre keine Wohltätigkeitsorganisation, sondern ein gewinnorientiertes Unternehmen. Wenn man sich nicht an die Spielregel halten wolle, müsste man sich eine andere Plattform suchen. YouTube ist unangefochtener Marktführer als Online-Video-Portal. Es gibt kaum andere Video-Plattformen, die an die Relevanz von YouTube heranreichen könnten und somit eine Alternative böten. Hier zeigt sich deutlich ein neoliberales Motiv (hier ist der kritische Gebrauch dieses Begriffes beabsichtigt z.B. nach Chomsky (2000) gemeint).

Das neoliberale Subjekt seiner selbst ist nicht fähig $\mathrm{zu}$ Beziehung zu anderen, die frei vom Zweck wären. Zwischen Unternehmern entsteht auch keine zweckfreie Freundschaft. Frei-sein bedeutet aber ursprünglich bei Freunden sein. Freiheit und Freund haben im Indogermanischen dieselben Wurzeln. [...] Die totale Vereinzelung, zu der das neoliberale Regime führt, macht uns nicht wirklich frei. (Han 2014, S. 11).

$24<$ https://web.archive.org/web/20210722134751/https://www. youtube.com/intl/de/about/> Anm.: Die Inhalte der vier Wertesäulen wurden 2021 von der Webseite entfernt und durch andere Inhalte ersetzt. Hier verwende ich eine Archivversion der Webseite. [22.10.2021].
Nach Han steckt die Freiheit in der Krise. Das Subjekt wähnt sich frei, ist aber unfrei, da es sich selbst innere Zwänge, wie Leistungs- und Optimierungszwang auferlegt. Depression und Burnout sind für Han ,,der Ausdruck einer tiefen Krise der Freiheit“ (ebd. S. 10). Burnout hängt mit der Arbeitsform und dem System zusammen.

In einem Video $^{25}$ zum Thema Creator-Burnout auf dem YouTube eigenen Kanal YouTube Creators wird Elle Mills, die 2018 über ihren eigenen Burnout mit nur 19 Jahren berichtet hatte, gegen Ende des Videos vom Interviewleiter gefragt: „(...) but is there anything that we should do to help with this issue as a platform?" Auf diese geschlossene Frage, verneint Elle Mills nach kurzem Zögern die Frage und meint, dass es von YouTubes Seite ausreiche über das Thema zu sprechen. Gegen Ende des Videos erklärt Elle Mills auf die Frage, wie es ihr besser gehen könnte: „A lot of it is a“,me“-problem, and I think that's working on myself and taking the time to work on myself. And getting extra help, like therapy obviously.,.. Elle Mills gibt vor, keine Verantwortung des Systems oder der Organisation YouTube zu sehen. Burnout ist für sie ein individualisiertes Problem. YouTube schlägt YouTuber:innen vor, wenn sie erste Anzeichen eines Burnouts bemerken würden, eine Auszeit von YouTube und professionelle Hilfe in Anspruch zu nehmen ${ }^{26}$. Vorschläge wie der damit verbundene Arbeitsausfall (Existenzangst) und die Kosten für z. B. therapeutische Hilfe o. ä. ausgeglichen werden können, gibt es bislang nicht.

YouTube sieht die Creator zwar als einen von drei Bausteinen seines Systems und betont, welche Wichtigkeit die YouTuber:innen für die Organisation haben. Nachhaltige Verantwortung, wie zum Beispiel eine firmeninterne Notfallhotline oder dem zu Verfügung stellen von professioneller Hilfe, wird momentan, soweit dies für Außenstehende einsehbar ist, nicht übernommen. Burnout ist nicht nur ein individuelles Problem. Bei der Burnout-Behandlung werden zwei Ansätze, der personenzentrierte und der organisationszentrierte Ansatz, zur nachhaltigen Verbesserung der Situation für Arbeiter:innen vorgeschlagen. Der personenzentrierte Ansatz beschäftigt sich mit dem“,,ausgebrannten oder Burnout-gefährdeten Individuum“ und beim organisationszentrierten Ansatz ,liegt der Schwerpunkt auf der Organisation von Arbeit und den Implikationen betrieblich initiierter Organisationsentwicklungsprozesse." (Moosbrugger 2012, S. 26). Jedoch gibt es zwischen YouTuber:innen und YouTube kein betrieblichen Vertragsverhält-

\footnotetext{
25 YouTube Creators: „Elle Mills zum YouTuber-Burnout I Creator Coffees" vom 05.02.2019 < https://www.youtube.com/watch? $\mathrm{v}=$ seCudUlxehs $>$ [24.05.2019].

26 YouTube Creators Academy: „Auf das eigene Wohlbefinden achten und Burn-out vermeiden" vom<https://creatoracademy.youtube.com/ page/course/well-being $>$ [25.04.20].
} 
nis, was einem Arbeitsvertrag z.B. mit Fürsorgepflichten des Arbeitgebers gleicht. Polemisch gefragt; wieso sollte YouTube also Verantwortung für die Arbeitsgesundheit von YouTuber:innen übernehmen?

Die Unsicherheit, die mit der Abhängigkeit von YouTube-Algorithmen und anderen Faktoren wie z. B. Algorithmus-Änderungen einhergeht, wurde von den Befragten erwähnt. Solche Änderungen und deren Folgen sind für die YouTuber:innen unberechenbar. Mögliche existenzielle Sorgen wurden von den Befragten am meisten in einer hauptberuflichen Tätigkeit gesehen. In der hauptberuflichen Tätigkeit auf YouTube ist es ratsam, mehrere Standbeine, z.B. auf anderen Plattformen wie Instagram, TikTok oder Twitch zu haben. Während der Diskussion um die Urheberrechtsreform 2018 wurden vor allem existentielle Sorgen in Videos von verschiedenen YouTuber:innen zu diesem Thema erwähnt.

\section{Fazit und Ausblick}

Im Frühjahr 2018 gründete der YouTuber Jörg Sprave (Küchemann 2019) die Gruppierung „YouTubers Union“, die seit dem Sommer 2019 mit der IG Metall im Projekt FairTube ${ }^{27}$ zusammenarbeitet. Mittlerweile ist daraus ein gemeinnütziger Verein entstanden, der auch international bessere Arbeitsbedingungen und Mitbestimmung für Content Creator fordert. Die „YouTubers Union“ ist keine legitime Gewerkschaft und kann demnach keine Mitgliedschaften ausstellen und sich auch nicht durch Mitgliedbeiträge finanzieren - was vor allem daran liegt, dass YouTuber:innen auf der ganzen Welt arbeiten. Es wäre denkbar, dass YouTuber:innen in jedem Land eine Gewerkschaft gründen, was aber wiederum einen immensen organisatorischen Aufwand bedeuten würde, würde man z.B. rechtlich gegen die Google LCC vorgehen wollen. YouTuber:innen sind selbstständig und nicht bei Google angestellt, jedoch hält YouTube das Monopol als OnlineVideoplattform, woraus eine besondere Verantwortung für Content Creator und Nutzer:innen hervorgehen sollte.

Fest steht, dass der Einfluss der Organisation YouTube, mit ihren systemimmanenten Algorithmen auf Content Creator enorm stark ist. Auch der Zeit-, Anforderungs- und Produktionsdruck (Pressure to Create) hat in den letzten Jahren mit den Veränderungen der Algorithmen zugenommen hat. Konträr hingegen verhalten sich undurchsichtige Strukturen, auf deren Veränderung YouTuber:innen im Grunde nur reagieren können und die ein Gefühl von völliger Machtlosigkeit auslösen können. Welchen Einfluss hat es auf die Arbeitsgesundheit von YouTuber:innen, wenn

$27<$ https://fairtube.info/> [22.10.2021]. diese selbst nur sehr wenige Hebel haben, die in Bewegung gesetzt könnten, um gehört und erst genommen zu werden?

Anfang November 2021 gab YouTube bekannt ${ }^{28}$, dass es die Anzeige von Dislikes von der Seite entfernen, um die psychische Gesundheit von YouTuber:innen bei möglichen koordinierten Shitstorm-Attacken zu schützen. Viele Creator ${ }^{29}$ sprechen sich gegen eine solche Änderung aus und fordern an Stelle einer globalen Änderung, die Entwicklung von Werkzeugen, die für diesen spezifischen Fall funktionieren, da das Like-Dislike-Verhältnis eine wichtige Grundlage für die Interaktion von Usern auf der Plattform ist.

Auch hier möchte ich die Vereinzelungstendenzen in digitalen Berufen hervorheben, die ich bereits im vorangegangenen Artikel (Albiez 2020) aufgeführt habe. Diese Tendenzen verstärken sich dadurch, dass das soziale System Gruppe z.B. auf YouTube eher weniger systemimmanent ist, sondern dass das Individuum und die Organisation sich gegenüberstehen. Inwiefern dies auf andere Bereiche und Organisationen zutrifft gilt es weiterhin zu untersuchen. Es stellt sich außerdem die Frage, welche Rolle Gruppe auf Plattformen überhaupt spielt, die eher dazu dienen Personen auszustellen?

In der Zukunft wird dieser Aspekt höchstwahrscheinlich mehr Bedeutung in der arbeitsweltlichen Beratung von Arbeiter:innen in digitalen Arbeitswelten haben. Der Faktor Gruppe und Gruppendynamik in digitalen Arbeitsfeldern wird allein durch eine fehlende körperlich-räumliche Erfahrung von Gruppe anders wahrgenommen und folgt mit hoher Wahrscheinlichkeit anderen Prinzipien, die es in $\mathrm{Zu}$ kunft zu erforschen gilt.

Funding Open Access funding enabled and organized by Projekt DEAL.

Open Access Dieser Artikel wird unter der Creative Commons Namensnennung 4.0 International Lizenz veröffentlicht, welche die Nutzung, Vervielfältigung, Bearbeitung, Verbreitung und Wiedergabe in jeglichem Medium und Format erlaubt, sofern Sie den/die ursprünglichen Autor(en) und die Quelle ordnungsgemäß nennen, einen Link zur Creative Commons Lizenz beifügen und angeben, ob Änderungen vorgenommen wurden.

Die in diesem Artikel enthaltenen Bilder und sonstiges Drittmaterial unterliegen ebenfalls der genannten Creative Commons Lizenz, sofern sich aus der Abbildungslegende nichts anderes ergibt. Sofern das betreffende Material nicht unter der genannten Creative Commons Lizenz steht und die betreffende Handlung nicht nach gesetzlichen Vorschriften erlaubt ist, ist für die oben aufgeführten Weiterverwendungen des Materials die Einwilligung des jeweiligen Rechteinhabers einzuholen.

Weitere Details zur Lizenz entnehmen Sie bitte der Lizenzinformation auf http://creativecommons.org/licenses/by/4.0/deed.de.

\footnotetext{
$28<\quad$ https://blog.youtube/news-and-events/update-to-youtube/> [13.11.2021].

${ }^{29}$ Marques Brownlee: Dear YouTube! vom 11.11.2021 < https://www. youtube.com/watch?v=CaaJyRvvaq8 $>$ [13.11.2021].
} 


\section{Literatur}

Albiez, M. (2020). Systemische, soziale und emotionale Herausforderungen und Glücksmomente im beruflichen Alltag von hauptund nebenberuflichen YouTuber*innen. Gruppe. Interaktion. Organisation. Zeitschrift Für Angewandte Organisationspsychologie (GIO), 51(4), 457-468. https://doi.org/10.1007/s11612-02000534-w.

Bunz, M. (2012). Die stille Revolution: wie Algorithmen Wissen, Arbeit, Öffentlichkeit und Politik verändern, ohne dabei viel Lärm zu machen. Berlin: . http://d-nb.info/101217008X/04

Burgess, J., \& Green, J. (2009). The entrepreneurial vlogger: participatory culture beyond the professional-amateur divide. In P. Snickars \& P. Vonderau (Hrsg.), The YouTube reader. Stockholm: National Library of Sweden.

Chomsky, N. (2000). Profit over people: Neoliberalismus und globale Weltordnung (Dt. Erstau). Hamburg: Europa-Verlag.

Chun, W.H.K. (2016). Updating to remain the same: habitual new media. : MIT Press.

Elsaesser, T. (2009). Tales of epiphany and entropy: around the worlds in eighty clicks. In P. Snickars \& P. Vonderau (Hrsg.), The YouTube reader. Stockholm: National Library of Sweden.

Han, B.-C. (2014). Psychopolitik: Neoliberalismus und die neuen Machttechniken. Frankfurt am Main: . http://d-nb.info/105084895 $0 / 04$

Krachten, C., \& Hengholt, C. (2018). YouTube: Spaß und Erfolg mit Online-Videos. Heidelberg: dpunkt.verlag.

Küchemann, F. (2019). Youtube unter Druck - Ruf nach Mitbestimmung. Frankfuter Allgemeine Zeitung. https://www.faz.net/ aktuell/feuilleton/medien/youtuber-gewerkschaft-fairtube-setztyoutube-unter-druck-16352663.html (Erstellt: 27.08.). Zugegriffen: 15. Aug. 2021.

Marek, R. (2013). Understanding YouTube: Über die Faszination eines Mediums. Bielefeld: transcript. http://deposit.d-nb.de/cgi-bin/ dokserv?id=4208861\&prov=M\&dok_var=1\&dok_ext=htm

Moosbrugger, J. (2012). Subjektivierung von Arbeit: Freiwillige Selbstausbeutung: Ein Erklärungsmodell für die Verausgabungsbereit- schaft von Hochqualifizierten. Wiesbaden: . https://doi.org/10. 1007/978-3-531-18788-4.

Muckenhuber, J. (2014). Arbeit ohne Ende?: Zur Arbeitsrealität der,,neuen “Selbstständigen. Konstanz: . http://d-nb.info/104425 8543/04

Opresnik, M.O., \& Yilmaz, O. (2016). Die Geheimnisse erfolgreichen YouTube-Marketings: Von YouTubern lernen und Social Media Chancen nutzen. Berlin, Heidelberg: . https://doi.org/10.1007/ 978-3-662-50317-1.

Passig, K. (2013). Standardsituationen der Technologiekritik. Berlin. http://scans.hebis.de/HEBCGI/show.pl?31985127_toc.pdf. Zugegriffen: 15. Aug. 2021.

Rieder, B., Matamoros-Fernández, A., \& Coromina, Ò. (2018). From ranking algorithms to "ranking cultures": investigating the modulation of visibility in youtube search results. Convergence, 24(1), 50-68. https://doi.org/10.1177/1354856517736982.

Walsh, G., Deseniss, A., \& Kilian, T. (2020). Marketing: Eine Einführung auf der Grundlage von Case Studies. Berlin, Heidelberg: https://doi.org/10.1007/978-3-662-58941-0.

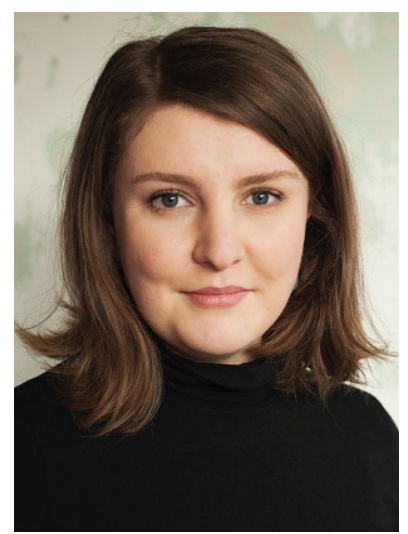

Milena Albiez Visuelle Kommunikation (M.F.A), Mehrdimensionale Organisationsberatung, Supervision und Coaching (M.A.) an der Universität Kassel. Sie arbeitet als künstlerische Mitarbeiterin an der Kunsthochschule Kassel und ist deutschlandweit als Supervisorin, Coach und Organisationsberaterin tätig. Sie ist ifag ${ }^{\circledR}$-zertifizierte Gruppendynamikerin und DGSv-zertifizierte Beraterin. www.milenaalbiez.de. 